\title{
Multiannual variability of spring discharge in Southern Poland
}

\author{
${ }^{1}$ Department of Hydrology and Water Management, University of Lodz, 90-139 Lodz, Narutowicza 88, pok. 203, Poland \\ ${ }^{2}$ Regional Inspectorate of Environmental Protection in Lodz, 90-743 Lodz, Lipowa 16, Poland; *Corresponding author, E-mail: piotr.mon- \\ iewski@geo.uni.lodz.pl
}

(Received: Nov 9, 2018; Revised accepted: May 29, 2019)

https://doi.org/10.18814/epiiugs/2019/019015

Discharge is one of the most important quantitative characteristics of springs. It not only specifies the yield of groundwater but also reflects the water retention conditions of the aquifer. An analysis of a long-term spring discharges enables linking them to hydrogeological and meteorological conditions of their catchment area as well as calculation of seasonal and multiannual variability parameters. The paper includes data on 84 springs in southern Poland collected in PIG-PIB databases. The time series are of different duration and were carried out in the period 1973-2016. The spring discharge variability index varied widely: from two to over two thousand, wherein the percentage of slightly variable, variable, and highly variable springs ranged around $33 \%$. The discharge of individual springs showed significant seasonal variations. The volume of outflowing water depended on current atmospheric conditions but it was primarily controlled by the hydrogeological type of springs and their location. Discharge of fissures springs as well as of low-flow outflows was generally more variable than that of porous or larger springs. Seasonality of discharge depended rather on the elevation of the spring than on its discharge - springs at higher altitudes had higher seasonality indices of discharge and a later date of its annual maximum discharge.

\section{Introduction}

Spring discharge depends on relatively stable hydrogeological properties of an aquifer and dynamically changing meteorological conditions. Aquifer type determines the amount of subterranean catchment recharge physical and chemical properties of water (temperature, $\mathrm{pH}$ and electrical conductivity) that affect infiltration capacity of the precipitation, i.e. the amount of water penetrating the Earth's crust. Retention of the aquifer depends also on its hydrostatic pressure that, together with temperature, determine the rate of water movements within the aquifer (Memon, 1995). The processes occurring in the subterranean catchment of a spring are manifested by the amount of water, recorded as an outflow of a certain discharge. Its long-term analysis allows for identification of those factors in the recharge area that have the greatest impact on transformation of precipitation signal (Fiorillo and Doglioni, 2010) and for determination of parameters of seasonal variability and multiannual discharge (Tarafdar, 2013).

Determination of hydrogeological characteristics of the springs requires an analysis of a homogeneous hydrological time-series of regular discharge measurements conducted over a multi-year period. Single or occasional measurements would not provide data, necessary to obtain characteristics fully reflecting the effects of meteorological conditions on the characteristics of the drained aquifer. Variability of discharge is not only an important indicator of aquifer structure (Diodato et al., 2017; Fiorillo and Guadagno, 2012) or a determinant of habitat conditions (Dumnicka et al., 2012), but also a marker of climatic trends (Fiorillo et al., 2015; Guo et al., 2005; Hao et al., 2015) or human impact on groundwater environment (Liu et al., 2017). Variability of discharge in selected springs of mountain and highland areas of Poland was investigated by Barczyk et al. (1999), Jokiel (1994, 1997), Barczyk (2003), Humnicki (2006, 2012, 2015), Satora et al. (2010), Moniewski (2015, 2016), Michalczyk et al. (2015) and in multi-year periods by Michalczyk et al. (2008), Michalczyk and Chabudziński (2013), Siwek (2013) as well as Siwek and Pociask-Karteczka (2017). However, not many reports have been published in this field, as accurate examination of spring discharge variability requires relatively long series of measurements with at least monthly data resolution. In addition, most of the papers concern only one or a few springs located close to each other. Therefore, the authors attempted to examine the yield variability of a larger group of springs located in southern Poland by using the available data. The area of their distribution covers the area of the Polish Carpathians and Sudetes.

\section{Materials and Methods}

This analysis is based on 84 discharge series recorded for the springs of southern Poland (Fig. 1), and stored in two related databases. The Groundwater Monitoring Database (GMD) covers 75 springs of which 56 are also represented in the Central Hydrogeological Data Bank (HYDRO Bank) (CBDH). In addition, 50 outflows of those listed in GMD are also registered as sites of Chemical Status Monitoring (CSM). Moreover, the HYDRO Bank expanded the number of monitored springs with nine outflows represented neither in GMD nor CSM. Both databases were made available in January 2017 by National Hydrogeological Service, a unit within the Polish Geological Institute - National 


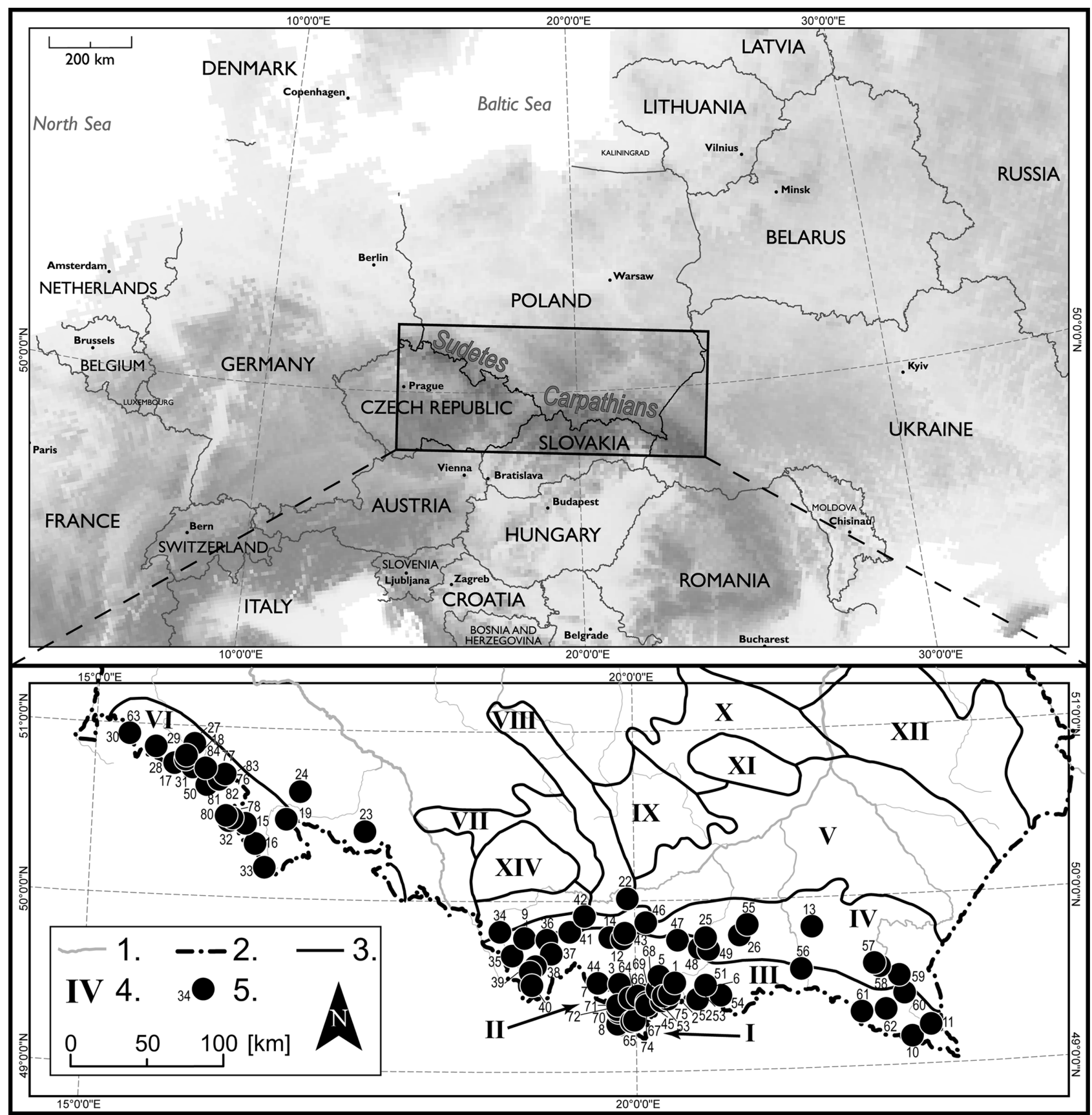

Figure 1. Localization of the studied springs in the south of Poland. Legend: 1 -rivers; 2 -border of Poland; 3 - borders of physico-geographical regions of Poland (simplified); 4-physico-geographical region number: I-The Tatra Mountains, II - The Podhale-Magura Area, III - The Beskids (Western, Middle and Eastern), IV - The Carpathian Foothills, V-The Subcarpathia, VI - The Sudetes (Western, Middle, and Eastern), VII - The Sudetic Foreland, VIII - The Wielun Upland, IX-The Nida Basin, X-The Krakow-Czestochowa Upland, XI - The Kielce Upland, XII - Lublin Upland and Roztocze, XIII - Przedborz, Upland, XVI - The Silesian Upland, XV - The Silesian Lowlands; 5 investigated springs (numbers compatible with Table 1). Source: own labor on the basis of PIG-PIB data.

Research Institute (PGI-NRI). The location of the investigated objects with reference to the geological structure and tectonics of the Polish area can be viewed using an interactive PGI-NRI website: http://geoportal.pgi.gov.pl

Majority of the springs were located in the mountain areas -27 springs in the Sudetes and 57 more to the east, in the Carpathians. The most commonly represented mesoregions of southern Poland (Solon et al., 2018) are the Western Beskids (21 springs), the Central Sudetes
(17), and the Podhale-Magura area (15). The lithological structure were dominated by shales with sandstones ( 32 springs), which together with sandstones (16), sands and shales made up $60 \%$ of the aquifer investigated area sediments (for the more complete information on a highly complicated stratigraphy of the southern Poland, please refer to the McCanns (2008a,b) study). The presence of carbonate rocks was confirmed for $19 \%$ of springs' catchments, and other components of the aquifers were magmatic rocks $(15 \%)$ and clastic rocks $(6 \%)$. 
The greatest number of springs originated from Carpathian flysch rocks of Paleogene (35) and Cretaceous (15) period and the lowest from Pleistocene sediments (3).

In the Carpathians similar number of springs originated within massifs (30) and intermontane valleys (27), while in the Sudetes they mostly outflowed within the massifs ( 23 vs. 4 in valleys). Altitude of the investigated springs differed greatly - total median for all outflows was $530 \mathrm{~m}$ a.s.l. and the greatest number of springs were located between 500 and $600 \mathrm{~m}$ a.s.l. (26). Elevations of the outflows ranged between 227 and $965 \mathrm{~m}$ a.s.l., and the lowest located spring was that in Kraków (No. 22). The springs within the Podtatrze and the Tatras were located at high altitudes, over $700 \mathrm{~m}$ a.s.l., and two of them originating in the Chochołowska Valley were at $965 \mathrm{~m}$ a.s.l. Mean elevation of the Sudeten springs was $518.7 \mathrm{~m}$ a.s.1., and of the Carpathian ones 548.1 $\mathrm{m}$ a.s.l. In the Carpathians, average elevation of mountain and submountain outflows was very similar but the springs originating in depressions and valleys were characterized by a much wider range of elevations (Fig. 2). In the Sudetes, the differences between mountain and sub-mountain elevations were greater and their medians differed by $170 \mathrm{~m}$. A predominant variant in both groups were the springs located on mountain slopes, as only $47 \%$ of the Carpathian and $15 \%$ of the Sudeten springs were located within land depressions and sinkholes. Based on the lithological, hydrogeological and stratigraphic characteristics of the aquifer, the investigated outflows were classified into one of three basic hydrogeological categories (Alfaro and Wallace, 1994): fissure (76), porous (5), and karst (3) springs. This large disproportion of the number of the investigated karst and porous springs in relation to fissures is caused by the relatively rare occurrence of the former group in this area.

The time-series collected in GMD and $\mathrm{CBDH}$ databases were recorded in the period 1973-2016. Their duration ranged from a few months to almost 40 years, $43 \%$ of them being conducted for less than a decade. There are many years with incomplete data, especially in the initial or final part of the series. Their completeness per year in these cases does not reach 50\%. Rare are also a gaps in observations ranging from one to three years. However, as many as 19 time-series

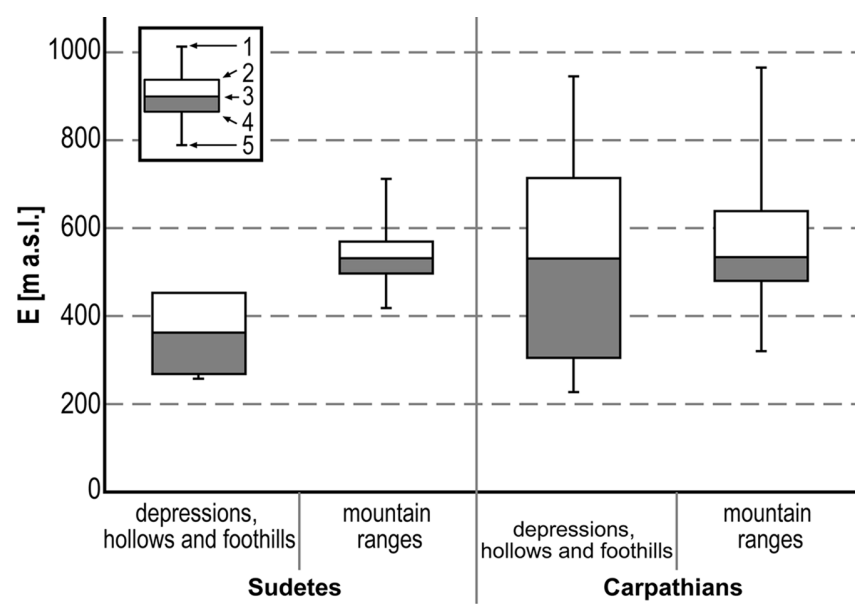

Figure 2. Elevation (E [m a.s.l.]) of the southern Poland springs. Legend: 1 - maximum $\left(E_{\text {max }}\right) ; 2$ - third quartile $\left(E_{Q 3}\right) ; 3$ - median $\left(E_{M e}\right) ; 4$ - first quartile $\left(E_{Q 1}\right) ; 5$ - minimum $\left(E_{\text {min }}\right)$. Source: own labor on the basis of PIG-PIB data. lasted for longer than 25 years. Discharge of the spring No. 65 in Zakopane 1 was observed for 31 years, and for the springs in Falsztyn (No. 4) and Dębno (No. 69) nearly complete time-series were collected in the period of almost 40 years. The number of simultaneously observed springs ranged from 20 in the 1970 s to nearly 50 in the 1980s and approximately $30-40$ in the last decade. The more detailed analysis of the quality of the data was presented in the previously published paper of the authors (Bartnik and Moniewski, 2018).

The discharge of 78 springs have been measured regularly once a week (at Mondays). The measurements of the rest of them in some periods have been performed once per month. The discharge of the very small springs was measured using the volumetric method (bucket technique). In the rest of them the $\mathrm{V}$-notch weirs with limnigraph have been used (but in this case in the database only the discharged from 6 UTC were registered).

The variability analysis included series of minimum, mean and maximum monthly discharges expressed in $\mathrm{dm}^{3} \cdot \mathrm{s}^{-1}$. They were obtained from time measurements performed with different intervals (usually once a week but some of them were measured daily and the others monthly). The databases also contain information about the location of the outflows, their elevation, and the lithographic and stratigraphic characteristics of the rocks forming their subterranean catchment. Analogical method was used to verify multiple zero discharges and only three temporarily disappearing springs were identified: in Rytro (No. 51), Sanok 1 (No. 57) and Zakopane 1 (No. 65). Further analyses (like e.g. seasonality measures) should included only the series without longer observation gaps (Katsanou et al., 2015). Therefore a few springs which series covering less than three years of continuous observations were removed. In the paper springs with measurement series covering at least three years of continuous observations were allowed. For the purpose of the data quality assessment, a column containing the number of months with discharge measurements of each spring was shown in Table 1.

Seasonal characteristics were calculated based on mean monthly discharges. For each month in which at least one measurement of discharge was made, the arithmetic mean of all data from that month was calculated. This solution was necessary considering variable times of measurements and resulting data asynchronicity (measurements on different days of the week).

Furthermore, different measures of dispersions were calculated (Kř́iž, 1973; Flora, 2004; Springer and Stevens, 2008; Kresic and Stevanovic, 2009):

$$
\begin{aligned}
& V=\frac{Q_{\max }-Q_{\min }}{Q_{m}} \cdot 100 \%, \\
& M=\frac{Q_{\max }}{Q_{\min }}, \\
& C_{V}=\frac{S D_{Q}}{Q_{m}},
\end{aligned}
$$

where $M, V, C_{V}$ are the coefficients of variation (according to Meinzer (1923), Maillete (1905) and Cv ratio, respectively), $Q_{\max }, Q_{m}, Q_{\min }$ are the maximum, mean and minimum monthly discharge $\left(\mathrm{dm}^{3} \cdot \mathrm{s}^{-1}\right)$ and $S D_{Q}$ is the standard deviation for mean monthly discharge $\left(\mathrm{dm}^{3} \cdot \mathrm{s}^{-1}\right)$.

Seasonal variability of discharge was analyzed using box and whiskers charts and so called Markham ratios based on mean monthly values (Markham, 1970). The seasonality power expressed by the seasonality 
Table 1. Parameters of the investigated spring discharge in the south of Poland (numbers compatible with Fig. 1)

\begin{tabular}{|c|c|c|c|c|c|c|c|c|c|c|c|c|c|c|}
\hline Figure & Localization & $\mathrm{E}$ & $S$ & $\mathrm{~L}$ & $\mathrm{~T}$ & $\mathrm{~N}_{\mathrm{m}}$ & $\mathrm{Q}_{\mathrm{m}}$ & $\mathrm{C}_{\mathrm{V}}$ & V & $\mathrm{M}$ & $\mathrm{C}_{\mathrm{A}}$ & $\mathrm{C}_{\mathrm{E}}$ & SI & $\mathrm{CT}$ \\
\hline 1 & Ochotnica Dolna & 557.5 & $\operatorname{Pg}(\mathrm{E}+\mathrm{Ol})$ & $\mathrm{sl}+\mathrm{pc}$ & $\mathrm{F}$ & 480 & 0.16 & 0.86 & 480 & 89 & 1.57 & 3.35 & 24.1 & 18.IV \\
\hline 2 & Czorsztyn & 502.5 & $\mathrm{Pg}(\mathrm{E}+\mathrm{Ol})$ & $\mathrm{sl}+\mathrm{pc}$ & $\mathrm{F}$ & 578 & 0.08 & 0.83 & 578 & 66 & 3.57 & 16.93 & 21.4 & 29.IV \\
\hline 3 & Pyzówka & 737.5 & $\mathrm{Pg}(\mathrm{E}+\mathrm{Ol})$ & ss & $\mathrm{F}$ & 348 & 0.21 & 0.58 & 348 & 25 & 2.05 & 6.87 & 7.8 & $21 . \mathrm{V}$ \\
\hline 4 & Falsztyn & 647.5 & $\mathrm{~J} 2+\mathrm{K} 1$ & ls & $\mathrm{F}$ & 274 & 0.94 & 0.60 & 274 & 31 & 0.58 & -0.64 & 17.6 & 17.IV \\
\hline 5 & Ochotnica Górna & 715.0 & $\mathrm{~K} 1$ & is & $\mathrm{F}$ & 181 & 0.26 & 0.47 & 181 & 32 & 1.45 & 0.98 & 7.6 & $10 . \mathrm{V}$ \\
\hline 6 & Jaworki & 650.0 & $\mathrm{~J} 2$ & ls & $\mathrm{F}$ & 362 & 0.14 & 0.53 & 362 & 4 & 2.63 & 10.23 & 11.8 & 19.VI \\
\hline 7 & Zubrzyca Dolna 1 & 642.5 & $\mathrm{Pg}(\mathrm{E}+\mathrm{Ol})$ & ss & $\mathrm{F}$ & 181 & 0.07 & 0.29 & 181 & 11 & 1.26 & 3.45 & 5.3 & $11 . \mathrm{V}$ \\
\hline 8 & Dolina Chochołowska 1 & 965.0 & $\mathrm{~T} 2$ & do & $\mathrm{K}$ & 119 & 241.0 & 0.30 & 119 & 5 & -1.05 & 0.93 & 7.9 & 11.XI \\
\hline 9 & Bielsko-Biała & 502.0 & $\mathrm{~K} 2$ & ss & $\mathrm{F}$ & 246 & 0.30 & 0.62 & 246 & 7 & 0.29 & -0.36 & 12.0 & $5 . \mathrm{IV}$ \\
\hline 10 & Wetlina & 694.0 & $\mathrm{PgOl}$ & $\mathrm{ss}+\mathrm{sl}$ & $\mathrm{F}$ & 267 & 0.26 & 0.52 & 267 & 50 & 0.88 & 0.79 & 16.8 & 23.III \\
\hline 11 & Dwerniczek & 565.0 & $\mathrm{PgOl}$ & SS & $\mathrm{F}$ & 1273 & 0.42 & 0.94 & 1273 & 32 & 7.71 & 88.59 & 18.0 & $4 . \mathrm{V}$ \\
\hline 12 & Zawadka 1 & 600.0 & $\mathrm{Pg}$ & ss & $\mathrm{F}$ & 625 & 0.21 & 1.11 & 625 & 80 & 2.61 & 8.46 & 33.3 & 14.IV \\
\hline 13 & Widacz & 242.0 & $\mathrm{Pg}$ & $\mathrm{ss}+\mathrm{sl}$ & $\mathrm{F}$ & 272 & 0.09 & 0.57 & 272 & 120 & 1.36 & 1.96 & 12.4 & $10 . \mathrm{VI}$ \\
\hline 14 & Bieńkówka & 550.0 & $\mathrm{Pg}$ & $\mathrm{ss}+\mathrm{sl}$ & $\mathrm{F}$ & 248 & 0.24 & 0.60 & 248 & 9 & 0.45 & -0.59 & 16.0 & 28.III \\
\hline 15 & Szczytna & 478.0 & $\mathrm{~K} 2$ & me & $\mathrm{F}$ & 98 & 10.06 & 0.18 & 98 & 60 & -0.04 & -0.24 & 1.9 & 4.IV \\
\hline 16 & Młoty & 521.0 & $\mathrm{~K} 2$ & me & $\mathrm{F}$ & 536 & 1.72 & 0.67 & 536 & 3 & 3.29 & 14.93 & 7.1 & 12.IV \\
\hline 17 & Kowary 1 & 542.0 & $\mathrm{C} 3$ & $\{\mathrm{~g}\}$ & $\mathrm{F}$ & 953 & 0.33 & 0.94 & 953 & 27 & 5.25 & 38.42 & 19.8 & $30 . \mathrm{VI}$ \\
\hline 18 & Kowary 2 & 576.0 & $\mathrm{C} 3$ & $\{\mathrm{~g}\}$ & $\mathrm{F}$ & 724 & 0.68 & 1.84 & 724 & 30 & 2.67 & 6.08 & 43.8 & 3.VIII \\
\hline 19 & Laski & 458.0 & $\mathrm{C} 3$ & (g) & $\mathrm{F}$ & 222 & 0.57 & 0.40 & 222 & 58 & 1.46 & 3.55 & 11.2 & 7.IV \\
\hline 20 & Kowalowa & 626.0 & $\mathrm{P}$ & $\mathrm{tt}+\mathrm{tf}$ & $\mathrm{F}$ & 751 & 4.21 & 0.98 & 751 & 5 & 2.44 & 9.25 & 20.2 & 7.IV \\
\hline 21 & Dobromyśl & 553.0 & $\mathrm{~K} 2$ & ss & $\mathrm{F}$ & 1082 & 1.47 & 1.17 & 1082 & 215 & 4.62 & 31.18 & 18.9 & 4.IV \\
\hline 22 & Kraków & 226.8 & $\mathrm{~J} 3$ & $\mathrm{w}$ & $\mathrm{K}$ & 158 & 1.15 & 0.38 & 158 & 406 & 0.76 & 0.56 & 15.3 & 24.IV \\
\hline 23 & Rudziczka & 258.0 & Q & $\mathrm{sd}+\mathrm{gr}$ & $\mathrm{P}$ & 73 & 1.64 & 0.16 & 73 & 5 & 0.63 & 0.06 & 1.3 & 9.III \\
\hline 24 & Czerńczyce & 272.0 & Q & sd+gr & $\mathrm{P}$ & 52 & 0.52 & 0.11 & 52 & 2 & 1.44 & 1.33 & 0.6 & 27.III \\
\hline 25 & Rożnów & 255.0 & $\mathrm{Pg}$ & ss & $\mathrm{F}$ & 90 & 0.11 & 0.25 & 90 & 2 & 0.65 & -0.74 & 9.7 & 22.VIII \\
\hline 26 & Ciężkowice & 257.0 & $\mathrm{Pg}$ & Ss & $\mathrm{F}$ & 201 & 0.02 & 0.56 & 201 & 2 & 1.12 & 0.28 & 28.8 & $16 . \mathrm{VI}$ \\
\hline 27 & Janowice & 418.0 & $\mathrm{C} 3$ & $\{\mathrm{~g}\}$ & $\mathrm{F}$ & 337 & 1.36 & 0.43 & 337 & 6 & 1.94 & 8.72 & 10.2 & 30.IV \\
\hline 28 & Karpacz & 712.0 & $\mathrm{C} 3$ & $\{\mathrm{~g}\}$ & $\mathrm{F}$ & 918 & 0.092 & 0.75 & 918 & 10 & 5.25 & 54.61 & 12.4 & $28 . \mathrm{V}$ \\
\hline 29 & Górzyniec & 468.0 & $\mathrm{C} 3$ & (h) & $\mathrm{F}$ & 249 & 0.21 & 0.74 & 249 & 2214 & 2.28 & 4.96 & 27.3 & $8 . \mathrm{VI}$ \\
\hline 30 & Czerniawa - Zdrój 1 & 453.0 & $\mathrm{Pt}$ & $\mathrm{sl}$ & $\mathrm{F}$ & 528 & 4.26 & 0.74 & 528 & 5 & 1.25 & 3.56 & 13.7 & 24.III \\
\hline 31 & Paczyn & 699.0 & $\mathrm{O}+\mathrm{S}$ & (a) & $\mathrm{F}$ & 261 & 0.33 & 0.55 & 261 & 303 & 0.84 & 1.25 & 18.7 & 21.III \\
\hline 32 & Jakubowice & 571.0 & $\mathrm{C}$ & $\{g\}$ & $\mathrm{F}$ & 383 & 0.28 & 0.79 & 383 & 14 & 1.18 & 1.90 & 37.1 & 13.III \\
\hline 33 & Różanka & 522.0 & $\mathrm{Pt}$ & $\mathrm{sl}$ & $\mathrm{F}$ & 405 & 0.40 & 0.73 & 405 & 37 & 1.75 & 3.12 & 12.3 & 26.IV \\
\hline 34 & Skoczów & 325.0 & $\mathrm{~J} 3$ & ls & $\mathrm{F}$ & 223 & 0.17 & 0.82 & 223 & 14 & 0.99 & -0.75 & 52.4 & 13.XI \\
\hline 35 & Ustroń & 500.0 & $\mathrm{~K} 2$ & $\mathrm{ss}+\mathrm{sl}$ & $\mathrm{F}$ & 684 & 0.67 & 0.85 & 684 & 12 & 2.73 & 12.34 & 18.8 & 13.IV \\
\hline 36 & Czernichów & 370.0 & $\mathrm{~K} 2$ & $\mathrm{ss}+\mathrm{sl}$ & $\mathrm{F}$ & 604 & 0.38 & 0.85 & 604 & 151 & 2.07 & 6.63 & 16.8 & 26.III \\
\hline 37 & Żywiec & 508.3 & $\mathrm{PgPc}$ & $\mathrm{ss}+\mathrm{sl}$ & $\mathrm{F}$ & 496 & 0.16 & 0.89 & 496 & 232 & 1.56 & 3.31 & 16.3 & $2 . \mathrm{V}$ \\
\hline 38 & Węgierska Górka & 442.2 & $\mathrm{PgPc}$ & $\mathrm{ss}+\mathrm{sl}$ & $\mathrm{F}$ & 462 & 0.58 & 1.01 & 462 & 81 & 1.76 & 3.52 & 37.3 & 21.III \\
\hline 39 & Kamesznica & 496.5 & $\mathrm{PgOl}$ & $\mathrm{ss}+\mathrm{sl}$ & $\mathrm{F}$ & 525 & 1.37 & 0.81 & 525 & 135 & 2.16 & 6.76 & 16.8 & 3.III \\
\hline 40 & Sól & 540.5 & $\mathrm{PgE}$ & $\mathrm{ss}+\mathrm{sl}$ & $\mathrm{F}$ & 192 & 0.62 & 0.60 & 192 & 285 & 0.28 & -0.97 & 36.0 & $6 . I V$ \\
\hline 41 & Ponikiew & 538.5 & $\mathrm{~K} 2$ & $\mathrm{ss}+\mathrm{co}+\mathrm{sl}$ & $\mathrm{F}$ & 1143 & 0.14 & 1.03 & 1143 & 14 & 4.31 & 33.66 & 14.6 & $1 . \mathrm{VI}$ \\
\hline 42 & Babica & 289.4 & $\mathrm{~K}$ & $\mathrm{ss}+\mathrm{sl}$ & $\mathrm{F}$ & 197 & 0.29 & 0.32 & 197 & 311 & 0.80 & 1.18 & 3.7 & 19.VI \\
\hline 43 & Stróża & 320.0 & $\mathrm{Pg}(\mathrm{E}+\mathrm{Ol})$ & $\mathrm{ss}+\mathrm{sl}$ & $\mathrm{F}$ & 228 & 0.05 & 0.33 & 228 & 7 & 1.91 & 6.19 & 8.4 & 30.III \\
\hline 44 & Zubrzyca Dolna 2 & 642.0 & $\mathrm{PgE}$ & $\mathrm{ss}+\mathrm{sl}$ & $\mathrm{F}$ & 114 & 0.07 & 0.23 & 114 & 6 & 0.89 & 0.77 & 6.8 & $23 . \mathrm{IV}$ \\
\hline 45 & Białka Tatrzańska 1 & 725.0 & $\mathrm{PgOl}$ & $\mathrm{ss}+\mathrm{sl}$ & $\mathrm{F}$ & 135 & 0.20 & 0.24 & 135 & 5 & 0.95 & 1.76 & 5.5 & 1.VI \\
\hline
\end{tabular}


Table 1. Continued

\begin{tabular}{|c|c|c|c|c|c|c|c|c|c|c|c|c|c|c|}
\hline Figure & Localization & $\mathrm{E}$ & $\mathrm{S}$ & $\mathrm{L}$ & $\mathrm{T}$ & $\mathrm{N}_{\mathrm{m}}$ & $\mathrm{Q}_{\mathrm{m}}$ & $\mathrm{C}_{\mathrm{V}}$ & $\mathrm{V}$ & $\mathrm{M}$ & $\mathrm{C}_{\mathrm{A}}$ & $\mathrm{C}_{\mathrm{E}}$ & SI & $\mathrm{CT}$ \\
\hline 46 & Czasław & 295.0 & $\mathrm{~K} 2$ & $\mathrm{ss}+\mathrm{sl}$ & $\mathrm{F}$ & 208 & 0.04 & 0.49 & 208 & 3 & 0.96 & 0.45 & 10.6 & 16.VI \\
\hline 47 & Młynne & 425.0 & $\mathrm{PgE}$ & $\mathrm{ss}+\mathrm{sl}$ & $\mathrm{F}$ & 614 & 0.33 & 0.76 & 614 & 10 & 2.28 & 9.27 & 17.5 & $14 . \mathrm{V}$ \\
\hline 48 & Zawadka 2 & 530.0 & $\mathrm{PgE}$ & $\mathrm{sl}+\mathrm{ss}$ & $\mathrm{F}$ & 406 & 0.44 & 0.57 & 406 & 59 & 1.16 & 3.32 & 17.0 & 9.VII \\
\hline 49 & Zbyszyce & 380.0 & $\mathrm{PgOl}$ & $\mathrm{ss}+\mathrm{sl}$ & $\mathrm{F}$ & 211 & 0.26 & 0.43 & 211 & 215 & 0.94 & 1.00 & 14.4 & $29 . \mathrm{V}$ \\
\hline 50 & Uniemyśl & 531.6 & $\mathrm{~T}$ & SS & $\mathrm{F}$ & 159 & 2.76 & 0.48 & 159 & 8 & 0.55 & -0.80 & 16.9 & 12.IV \\
\hline 51 & Rytro & 480.0 & $\mathrm{Pg}(\mathrm{E}+\mathrm{Ol})$ & $\mathrm{ss}+\mathrm{sl}$ & $\mathrm{F}$ & 1064 & 0.08 & 1.25 & 1064 & 5 & 4.99 & 32.09 & 11.1 & $16 . \mathrm{V}$ \\
\hline 52 & Jaworki 1 & 640.0 & $\mathrm{PgPc}$ & $\mathrm{sl}+\mathrm{sS}$ & $\mathrm{F}$ & 486 & 0.28 & 0.96 & 486 & periodic & 3.51 & 13.79 & 23.2 & 3.III \\
\hline 53 & Jaworki 2 & 630.0 & $\mathrm{~J} 2+\mathrm{K} 1$ & ls & $\mathrm{F}$ & 513 & 0.08 & 0.73 & 513 & 26 & 2.24 & 8.35 & 10.4 & 12.VI \\
\hline 54 & Wierchomla Wielka & 495.0 & $\mathrm{PgE}$ & $\mathrm{sl}+\mathrm{ss}$ & $\mathrm{F}$ & 309 & 0.76 & 0.41 & 309 & 62 & 1.66 & 6.68 & 4.9 & $28 . \mathrm{V}$ \\
\hline 55 & Jodłówka Tuchowska & 280.0 & $\operatorname{Pg}(\mathrm{Pc}+\mathrm{E})$ & Ss & $\mathrm{F}$ & 458 & 0.08 & 0.80 & 458 & 13 & 1.42 & 2.31 & 21.2 & 30.III \\
\hline 56 & Kąty & 350.0 & $\mathrm{PgOl}$ & $\mathrm{ss}+\mathrm{sl}$ & $\mathrm{F}$ & 171 & 0.09 & 0.31 & 171 & 37 & 0.03 & 0.31 & 7.5 & $22 . \mathrm{V}$ \\
\hline 57 & Sanok 1 & 315.0 & $\mathrm{~K} 2$ & $\mathrm{ss}+\mathrm{sl}$ & $\mathrm{F}$ & 505 & 0.05 & 1.41 & 505 & 8 & 1.72 & 1.95 & 49.6 & $10 . \mathrm{V}$ \\
\hline 58 & Sanok 2 & 340.0 & $\mathrm{PgOl}$ & $\mathrm{sl}+\mathrm{sS}$ & $\mathrm{F}$ & 322 & 0.22 & 0.58 & 322 & periodic & 1.51 & 1.86 & 6.2 & $24 . \mathrm{VI}$ \\
\hline 59 & Bezmiechowa Górna & 395.0 & $\mathrm{~K} 2+\mathrm{PgOl}$ & $\mathrm{sl}+\mathrm{me}$ & $\mathrm{F}$ & 171 & 0.73 & 0.39 & 171 & 11 & -0.05 & -0.65 & 11.6 & 27.III \\
\hline 60 & Zabrodzie & 408.0 & $\mathrm{PgOl}+\mathrm{NgM}$ & $\mathrm{ss}+\mathrm{sl}$ & $\mathrm{F}$ & 194 & 0.19 & 0.47 & 194 & 11 & 0.60 & -0.10 & 15.5 & $5 . I V$ \\
\hline 61 & Radoszyce & 515.0 & $\mathrm{PgOl}$ & $\mathrm{ss}+\mathrm{sl}$ & $\mathrm{F}$ & 299 & 0.69 & 0.64 & 299 & 10 & 0.51 & -0.36 & 19.0 & $23 . I I$ \\
\hline 62 & Bystre & 480.0 & $\mathrm{PgOl}$ & $\mathrm{ss}+\mathrm{sl}$ & $\mathrm{F}$ & 226 & 0.95 & 0.37 & 226 & 123 & 1.22 & 3.21 & 8.2 & 12.VII \\
\hline 63 & Czerniawa - Zdrój 2 & 453.0 & $\mathrm{Pt}$ & sl & $\mathrm{F}$ & 105 & 2.64 & 0.30 & 105 & 7 & -0.58 & 0.11 & 12.4 & 9.VI \\
\hline 64 & Maruszyna & 712.5 & $\mathrm{~J}+\mathrm{K}$ & SS & $\mathrm{F}$ & 343 & 0.09 & 0.75 & 343 & 63 & 1.31 & 1.04 & 17.6 & $28 . \mathrm{VI}$ \\
\hline 65 & Zakopane 1 & 907.5 & $\mathrm{PgE}$ & Is & $\mathrm{F}$ & 1126 & 18.76 & 0.92 & 1126 & 21 & 4.23 & 41.53 & 30.8 & $25 . \mathrm{VI}$ \\
\hline 66 & Szaflary & 656.0 & $\mathrm{~J}$ & ls & $\mathrm{F}$ & 271 & 0.12 & 0.42 & 271 & periodic & 1.49 & 2.77 & 7.3 & $17 . \mathrm{V}$ \\
\hline 67 & Zakopane 2 & 910.0 & $\mathrm{Pg}(\mathrm{E}+\mathrm{Ol})$ & Is & $\mathrm{F}$ & 144 & 17.41 & 0.12 & 144 & 19 & 4.28 & 39.08 & 2.9 & $15 . \mathrm{VIII}$ \\
\hline 68 & Białka Tatrzańska 2 & 715.0 & Q & gr & $\mathrm{P}$ & 135 & 0.23 & 0.24 & 135 & 4 & 0.95 & 1.76 & 5.5 & 1.VI \\
\hline 69 & Dębno & 530.7 & Q & sd+gr+pe & $\mathrm{P}$ & 645 & 10.43 & 0.60 & 645 & 3 & 2.86 & 18.59 & 12.1 & $20 . \mathrm{V}$ \\
\hline 70 & Dzianisz & 945.0 & $\mathrm{Pg}(\mathrm{E}+\mathrm{Ol})$ & $\mathrm{ss}+\mathrm{sl}$ & $\mathrm{F}$ & 378 & 1.70 & 0.67 & 378 & 46 & 1.41 & 2.72 & 25.2 & $1 . \mathrm{VII}$ \\
\hline 71 & Ciche & 767.5 & $\mathrm{Pg}(\mathrm{E}+\mathrm{Ol})$ & $\mathrm{sd}+\mathrm{gr}$ & $\mathrm{P}$ & 165 & 0.20 & 0.30 & 165 & 44 & 1.03 & 0.99 & 7.4 & $24 . \mathrm{V}$ \\
\hline 72 & Witów & 836.5 & $\mathrm{Pg}(\mathrm{E}+\mathrm{Ol})$ & $\mathrm{ss}+\mathrm{sl}$ & $\mathrm{F}$ & 265 & 0.30 & 0.59 & 265 & 5 & 0.66 & -0.11 & 14.6 & 12.VI \\
\hline 73 & Niedzica & 535.0 & $\mathrm{~K}+\mathrm{Q}$ & $\mathrm{sd}+\mathrm{sl}$ & $\mathrm{F}$ & 211 & 0.14 & 0.52 & 211 & 50 & 0.23 & -0.85 & 13.4 & $15 . \mathrm{V}$ \\
\hline 74 & Dolina Chochołowska 2 & 965.0 & $\mathrm{~T} 2$ & do & $\mathrm{K}$ & 373 & 17.15 & 0.86 & 373 & 20 & 1.49 & 2.32 & 34.0 & 8.VII \\
\hline 75 & Łapsze Niżne & 610.0 & $\mathrm{Pg}(\mathrm{E}+\mathrm{Ol})$ & $\mathrm{ss}+\mathrm{sl}$ & $\mathrm{F}$ & 252 & 0.20 & 0.56 & 252 & 90 & 0.71 & -0.14 & 6.4 & $6 . I V$ \\
\hline 76 & Kowalowa & 530.5 & $\mathrm{P}$ & $\mathrm{tf}$ & $\mathrm{F}$ & 312 & 2.26 & 0.62 & 312 & 13 & 1.57 & 2.64 & 14.7 & $30 . \mathrm{IV}$ \\
\hline 77 & Dobromyśl & 534.5 & K & Ss & $\mathrm{F}$ & 420 & 5.57 & 0.87 & 420 & 30 & 1.41 & 1.63 & 20.2 & 14.III \\
\hline 78 & Darnków & 560.4 & $\mathrm{~K}$ & ss & $\mathrm{F}$ & 343 & 0.70 & 0.64 & 343 & 95 & 1.23 & 1.88 & 20.9 & $5 . \mathrm{III}$ \\
\hline 79 & Łączna & 613.8 & $\mathrm{~K}$ & ss & $\mathrm{F}$ & 490 & 1.33 & 1.11 & 490 & 143 & 1.75 & 2.79 & 25.9 & 3.III \\
\hline 80 & Czermna & 457.8 & $\mathrm{~K}$ & SS & $\mathrm{F}$ & 518 & 0.28 & 1.23 & 518 & 149 & 2.12 & 3.77 & 3.3 & 9.III \\
\hline 81 & Uniemyśl & 531.6 & $\mathrm{~T}$ & ss & $\mathrm{F}$ & 219 & 4.16 & 0.47 & 219 & 86 & 0.68 & 0.34 & 9.8 & 22.IV \\
\hline 82 & Mieroszów & 507.8 & $P$ & $\mathrm{tf}$ & $\mathrm{F}$ & 252 & 2.06 & 0.63 & 252 & 9 & 0.85 & 0.07 & 24.3 & 15.III \\
\hline 83 & Unisław Śląski & 567.8 & $\mathrm{P}$ & $\mathrm{tf}$ & $\mathrm{F}$ & 284 & 1.23 & 0.55 & 284 & 22 & 1.66 & 2.87 & 7.6 & 23.II \\
\hline 84 & Krzeszów & 486.0 & $\mathrm{P}$ & $\{t\}$ & $\mathrm{F}$ & 191 & 15.25 & 0.29 & 191 & 25 & 1.44 & 5.47 & 4.2 & 22.III \\
\hline
\end{tabular}

Source: data provided by PIG-PIB and own labor based on PSH monitoring data.

Explanations: $E$ - elevation [m a.s.l.]; $S$ - stratigraphy: $\mathrm{Pt}$ - Proterozoic, $\mathrm{O}+\mathrm{S}$ - Ordovician, Silurian, $\mathrm{C} 3$ - Pennsylvanian, $\mathrm{P}-\mathrm{Permian}, \mathrm{T}-\mathrm{Triassic}$, T2 - Middle Triassic, J - Jurassic, J3 - Late Jurassic, K - Cretaceous, K1 - Early Cretaceous, K2 - Late Cretaceous, PgPc - Paleogene, Paleocene, $\mathrm{PgE}$ - Paleogene, Eocene, PgOl - Paleogene, Oligocene, Pg(E+Ol) - Paleogene (Eocene + Oligocene), NgM - Neogene, Miocene, Q - Quaternary; $L$ - lithology: $\{\mathrm{g}\}$ - Granite, $\{\mathrm{t}\}$ - Trachyte, (a) - Amphibolite, (g) - Gneiss, (h) - Hornfels, sd - Sand, gr - Gravel, sl - Slate, pe - Pebble, ss - Sandstone, ls - Limestone, do - Dolostone, me - Marl, co - conglomerate, tf- Tuff, tt-Tuffite; $T$ - type of springs: F - fissure, $\mathrm{K}$ - karst, $\mathrm{P}$ - porous; $N_{m}-$ number of months with measurements; $Q_{m}$ - mean spring discharge $\left[\mathrm{dm}^{3} \cdot \mathrm{s}^{-1}\right] ; C v-$ spring variability index; $V-$ Meinzer's discharge class [\%]; $M-$ Maillete's spring variability class; $C_{A}$ - asymmetry of discharge; $C_{E}-$ kurtosis of discharge; $S I$ - seasonality index of discharge [\%]; $C T-$ date of maximum discharge (day). 
index (SI) reflected dominant degree of discharge within a specific season and the time of maximum discharge during the year (CT) reflected the month with the greatest discharge (Bartnik and Tomaszewski, 2006; Moniewski, 2015). As the springs were observed in different years, adopting synchronous reference periods was impossible.

Seasonality index is expressed by the equation (Bartnik and Tomaszewski, 2006; Moniewski, 2015):

$$
S I=\frac{\left|\overrightarrow{R_{m}}\right|}{\sum_{n=1}^{12}\left|\overrightarrow{r_{n}}\right|} \cdot 100 \%
$$

where $S I$ is the seasonality index, $n$ is the number of the month $(1,2,3 \ldots 12), \vec{r}_{n}$ is the vector identified as a mean spring discharge in the next month $\left(\mathrm{dm}^{3} \cdot \mathrm{s}^{-1}\right), \overrightarrow{R_{m}}$ is a resultant vector determined based on 12 vectors as their geometric mean $\left(\mathrm{dm}^{3} \cdot \mathrm{s}^{-1}\right)$, which is expressed by the equation:

$$
\overrightarrow{R_{m}}=\sqrt{\left(\sum_{n=1}^{12}\left[\left|\vec{r}_{n}\right| \cdot \cos \left(\alpha_{n}\right)\right]\right)^{2}+\left(\sum_{n=1}^{12}\left[\left|\vec{r}_{n}\right| \cdot \sin \left(\alpha_{n}\right)\right]\right)^{2}}
$$

where $\alpha_{n}$ is the angle defining the direction of the vector $\vec{r}_{n}$.

The date of an resultant vector of the mean maximum discharge $(C T)$ is calculated by the formula:

$$
C T=\left(\operatorname{arctg} \frac{\sum_{n=1}^{12}\left|\vec{r}_{n}\right| \cos \left(\alpha_{n}\right)}{\sum_{n=1}^{12}\left|\vec{r}_{n}\right| \sin \left(\alpha_{n}\right)}\right) \cdot \frac{365}{360} \text {. }
$$

Moreover, parameters of discharge distribution were assessed for individual springs and skewness ratio $\left(C_{A}\right)$ :

$$
C_{A}=\frac{\mu_{3}}{S D_{Q}^{3}}
$$

and excess kurtosis ratio $\left(C_{E}\right)$ were calculated:

$$
C_{E}=\frac{n \cdot(n+1)}{(n-1) \cdot(n-2) \cdot(n-3)} \cdot \sum_{n=1}^{N}\left(\frac{Q_{i}-Q_{m}}{S D_{Q}}\right)^{4}-\frac{3 \cdot(n-1)^{2}}{(n-2) \cdot(n-3)},
$$

where $\mu_{3}$ is the third central moment, $n$ is the number of observations, $Q_{i}$ is the $i$-th discharge of a spring in the sample $\left(\mathrm{dm}^{3} \cdot \mathrm{s}^{-1}\right)$ and $Q_{m}$ is the mean discharge of a spring $\left(\mathrm{dm}^{3} \cdot \mathrm{s}^{-1}\right)$.

Seasonal and multi-year variability of discharge was performed for the available series duration, and the parameters that required synchronous data were calculated for shorter series. However, it should be remembered that some variability measures depend on the duration of the analyzed series. For example, multiannual variability of discharge according to Meinzer $(\mathrm{r}=0.40)$, as well as asymmetry ratio $(\mathrm{r}=0.29)$ and discharge kurtosis ratio $(\mathrm{r}=0.34)$ positively correlated with the number of months included in the time-series. This is because longer observation periods increased the chance of recording extreme values. Adoption of a shorter but uniform reference period for all, or even for the majority of outflows, was prevented by usually variable periods of operation of the measuring stations.

\section{Results}

The smallest springs were dominated by the Carpathian outflows, while the Sudeten ones were usually more abundant (Buczyński, 2017). More than a half (53\%) of the investigated objects were small springs with discharge ranging from 0.1 to $1.0 \mathrm{dm}^{3} \cdot \mathrm{s}^{-1}$ (sixth magnitude), and mean discharge of only $0.33 \mathrm{dm}^{3} \cdot \mathrm{s}^{-1}$. The outflows of this group were mainly located in the Carpathian depressions and submontane valleys and this indicated huge runoff dispersion. Runoff in the higher parts of the massifs was more ordered due to advanced erosion processes (Lajczak, 2012). The investigated population of springs included also 14 objects with discharge below $0.1 \mathrm{dm}^{3} \cdot \mathrm{s}^{-1}$ (seventh magnitude) and 17 objects with discharge range of $1.0-10.0 \mathrm{dm}^{3} \cdot \mathrm{s}^{-1}$ (fifth magnitude). The smallest object belonging to seventh magnitude was the spring in Ciężkowice (No. 26) with a mean discharge nearly $0.02 \mathrm{dm}^{3} \cdot \mathrm{s}^{-1}$. There were six larger springs with mean discharge exceeding $10 \mathrm{dm}^{3} \cdot \mathrm{s}^{-1}$ (fourth magnitude), and the largest one (third magnitude) was Wywierzysko Chochołowskie (No. 8) $-241 \mathrm{dm}^{3} \cdot \mathrm{s}^{-1}$ (Table 1).

The Maillete's discharge variability coefficient $(M)$ for constant springs varied within a wide range of 1.6 to over 2,000 . Out of 84 outflows, only the spring in Czerńczyce (No. 24) may be classified as the one with constant discharge $(M=1.6)$. Low variability of discharge $(\mathrm{M}<3)$ was also observed for the springs in Rudziczka (No. 23), Rożnowo (No. 25) and Szczytna (No. 15). As per our classification, the number of springs with low $(2<\mathrm{M}<10)$, standard $(10<\mathrm{M}<50)$ and high variability $(M>50)$ was evenly distributed and each group contained $33 \%$ of total outflows. The last group included an extremely variable spring in Górzyniec ( $M=2214$; No. 29) and three temporarily disappearing ones in Zakopane 1 (No. 65), Rytro (No. 51) and Sanok 2 (No. 58).

Employing Meinzer's variability index (V) yielded slightly different results. According to this criterion there were no permanent springs $(0$ $<\mathrm{V}<25 \%$ ), only four springs showed low variability $(25 \%<\mathrm{V}<100 \%)$, and as many as 80 outflows were highly variable ( $\mathrm{V}>100 \%)$. Still the most stable discharge was observed for sub-Sudeten Czerńczyce ( $\mathrm{V}=53 \%$ ), and the most variable ( $\mathrm{V}>100 \%)$ were the springs in Dwerniczek (No. 11), Ponikiew (No. 41), Zakopane 1 (No. 65), Dobromyśl (No. 77) and Rytro (No. 51).

Similar conclusions were reached when Pearson criterion was used - low variability $\left(0<\mathrm{C}_{\mathrm{V}}<0.2\right)$ was confirmed for four springs, average $\left(0.2<\mathrm{C}_{\mathrm{V}}<0.4\right)$ for 16 , high $\left(0.4<\mathrm{C}_{\mathrm{V}}<1.0\right)$ for 55 , very high $\left(1.0<\mathrm{C}_{\mathrm{V}}<1.5\right)$ for 8 and extremely high $\left(\mathrm{C}_{\mathrm{V}}>1.5\right)$ only one spring Kowary 2 (No. 18). Apart from those listed above, the springs of high variability were also those, located in Sanok 1 (No. 57) and Czermna (No. 80).

A comparison of discharge variability coefficient of the Sudeten and Carpathian springs revealed its decreasing dynamics towards the east, as mean variability coefficients of the Sudeten outflows were slightly higher $\left(\mathrm{C}_{\mathrm{V}}=0.66, \mathrm{M}=28.1, \mathrm{~V}=363 \%\right)$ than those in the Carpathians $\left(C_{V}=0.57, M=19.4, V=275 \%\right)$. However, there were differences within both groups - in the Sudetes the discharge in sub-mountain outflows was significantly less variable than in the mountains, whereas the Carpathian springs were more uniform (Fig. 3). The hydrogeological conditions were also important, the variability coefficients for the porous springs were 1.2 to 2.1 times lower than analogical measures calculated for karst outflows and from 2.3 to 7.5 times lower than for fissure springs. Regardless of the small representativeness of karst and porous springs, this confirmed the key role of aquifer structure in the transformation of precipitation signal to subsurface runoff.

A weak negative significant correlation was found between outflow discharge and its variability coefficients (Table 2 ). Discharge variability was slightly more $(\mathrm{r}=-0.22)$ dependent on its minimum level $\left(\mathrm{Q}_{\min }\right)$. This negative correlation confirmed that small springs have larger dis- 

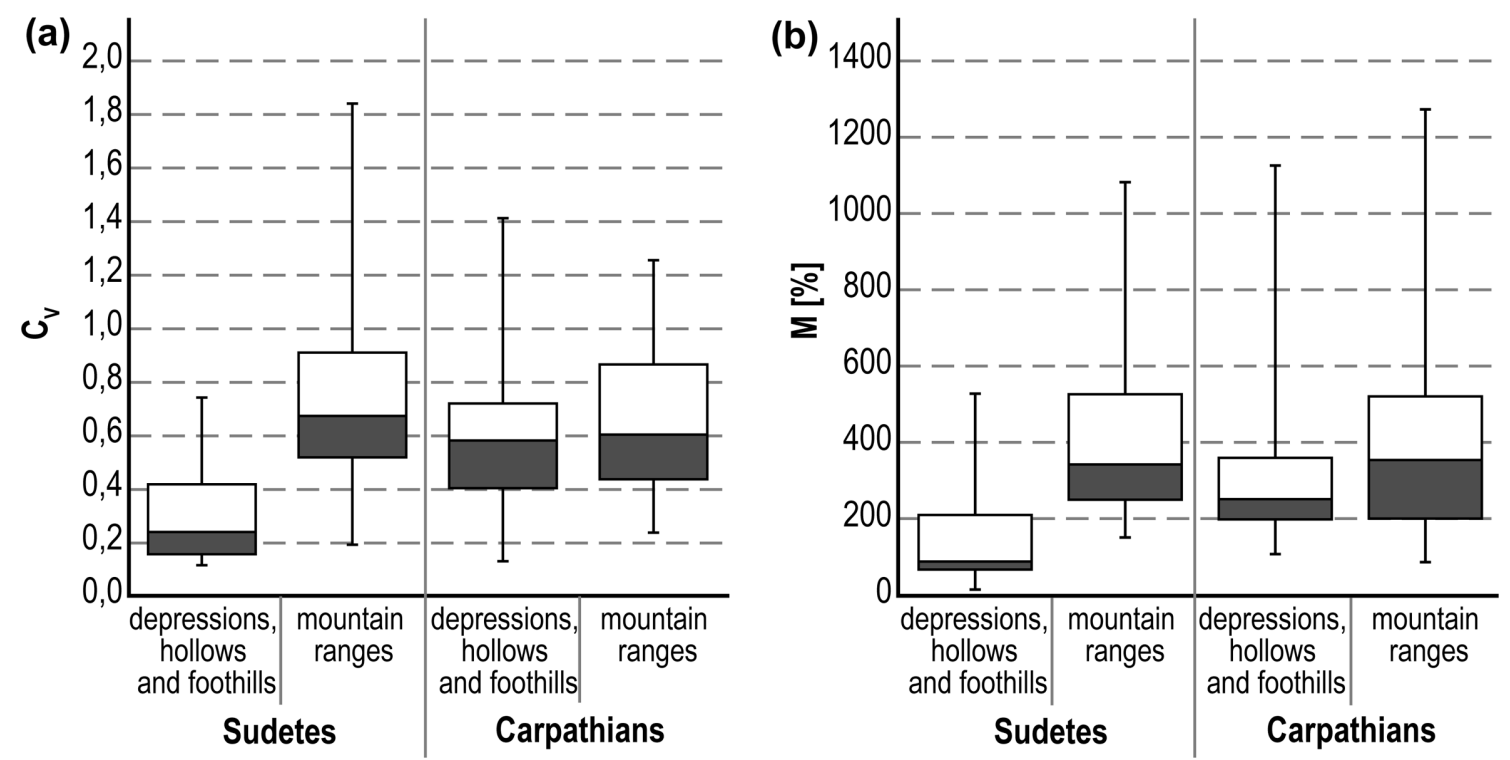

Figure 3. Main distribution characteristics of the spring discharge variability in the south of Poland: a - spring variability index $\left(C_{V}\right)$; $b-$ Meinzer's variability index (M) [\%]. Legend: 1-maximum; 2-third quartile; 3 -median; 4-first quartile; 5 -minimum. Source: own labor on the basis of PIG-PIB data.

Table 2. Pearson's correlation coefficients (r) between different South of Poland springs discharge characteristics (upper right part of matrix) and they $t$-Students Pearson's correlations significance test $(t-S)$ results (lower left part). The significant values $(\alpha=0.05$, df $=82)$ are indicated by bold

\begin{tabular}{|c|c|c|c|c|c|c|c|c|c|}
\hline${ }_{t-S}{ }^{r}$ & E & $\mathrm{Q}_{\mathrm{m}}$ & $\mathrm{C}_{\mathrm{V}}$ & V & M & $\mathrm{C}_{\mathrm{A}}$ & $\mathrm{C}_{\mathrm{E}}$ & SI & CT \\
\hline $\mathrm{E}$ & & $\mathbf{0 , 3 3}$ & 0,05 & 0,14 & $-0,10$ & 0,15 & 0,21 & 0,02 & 0,22 \\
\hline $\mathrm{Q}_{\mathrm{m}}$ & 3,16 & & $-0,13$ & $-0,10$ & $-0,05$ & $-0,19$ & $-0,02$ & $-0,08$ & 0,41 \\
\hline $\mathrm{C}_{\mathrm{V}}$ & 0,44 & $-1,20$ & & 0,74 & 0,05 & $\mathbf{0 , 5 1}$ & $\mathbf{0 , 3 0}$ & 0,67 & $-0,09$ \\
\hline V & 1,29 & $-\mathbf{- 0 , 8 8}$ & 9,95 & 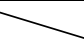 & $-0,06$ & $\mathbf{0 , 8 5}$ & 0,78 & 0,30 & $-0,05$ \\
\hline $\mathrm{M}$ & $-\mathbf{0 , 8 9}$ & $-0,43$ & $\mathbf{0 , 4 8}$ & $-0,57$ & & 0,02 & $-0,05$ & 0,13 & 0,01 \\
\hline $\mathrm{C}_{\mathrm{A}}$ & 1,38 & $-1,76$ & 5,30 & 14,50 & 0,16 & & 0,91 & 0,12 & 0,01 \\
\hline $\mathrm{C}_{\mathrm{E}}$ & 1,97 & $-0,16$ & 2,87 & 11,14 & $-0,46$ & 19,50 & & 0,01 & 0,10 \\
\hline SI & 0,19 & $-0,76$ & 8,13 & 2,87 & 1,17 & 1,07 & 0,13 & & 0,09 \\
\hline $\mathrm{CT}$ & 2,02 & 4,06 & $-0,84$ & $-0,45$ & 0,11 & 0,12 & 0,94 & $\mathbf{0 , 8 4}$ & \\
\hline
\end{tabular}

Source: own labor based on PSH monitoring data.

Explanations: as under the Table 1.

charge variability than the large ones, recharged from larger groundwater reservoirs. A correlation between variable discharge and outflow elevation doesn't exist $(r=0.05)$. This was due to the fact that the springs located at lower altitudes could drain the aquifer of greater thickness and their yield was less dependent on precipitation.

Variability of outflow discharges for which the time-series lasted longer than 36 months has also been analyzed (Table 1). The Markham's seasonality index (SI) changed from 0.6 (Czerńczyce) to nearly 50\% (a spring in Sanok). Although the seasonality index proved to be independent of the mean outflow discharge, a significant correlation was found between this measure and variability coefficients of Pearson ( $\mathrm{r}$ $=0.67)$ and Meinzer $(r=0.30)$. This means that the springs with higher discharge dynamics were also characterized by its higher seasonal variability. Contrary to that, outflows with stable yield experienced low seasonality.

Similar conclusions were reached after analyzing the metrics of discharge distribution. In majority of cases a symmetrical distribution or a slight positive skew $\left(\mathrm{C}_{\mathrm{A}}>0\right)$ were observed. Mean asymmetry coefficients for the discharge of the Sudeten and Carpathian springs were similar and reached $\mathrm{C}_{\mathrm{A}}=1.95$ and $\mathrm{C}_{\mathrm{A}}=1.68$, respectively. In the Sudetes, maximum excesses were slightly more frequent as those springs showed greater variability of discharge. Moreover, differences between mountain and sub-mountain outflows were visible in both groups, as the springs located at higher altitude demonstrated greater asymmetry and higher excess coefficient (Fig. 4).

A dominance of mesokurtic and leptokurtic distribution of spring discharge series, as well as a strong dominance of series, characterized by a positive skew were observed. Springs with high and very high variability experienced a greater number of outlying yields. Often the maximum recorded yields were associated with subsurface flow and sometimes surface runoff recharging the spring niches. In such cases their effects on the spring yield recorded in the gauging sections could 

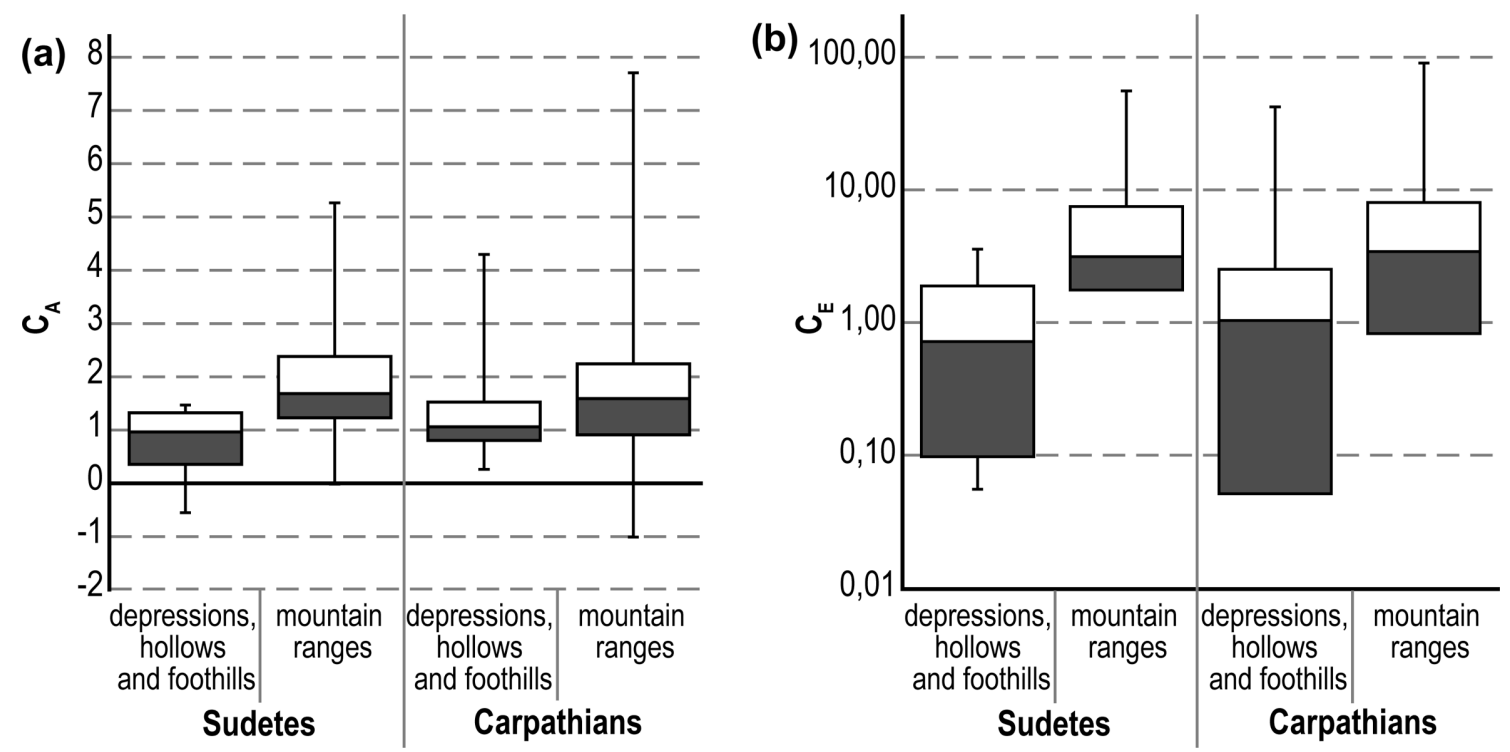

Figure 4. Main distribution characteristics of the spring discharge in the south of Poland: a-asymmetry $\left(C_{A}\right)$; b-excess kurtosis $\left(C_{E}\right)$. Legend: as in Fig. 2. Source: own labor on the basis of PIG-PIB data.

not be eliminated. The highest values of discharge distribution asymmetry $\left(\mathrm{C}_{\mathrm{A}}>5\right)$ were determined for the springs of very high variability in Dwerniczek (No. 11), Karpacz (No. 28) and Kowary 1 (No. 17). Both measures of distribution of outflow discharge series were characterized, for obvious reasons (mathematical interdependence - derivatives of central moments), by very strong direct linear correlation $(\mathrm{r}=0.91)$.

The time of CT was much more variable than the seasonality index. As the outflow yield depends on recharging of the drained groundwater reservoir, the peak discharges were observed in the spring during maximum water recharge to the aquifer (Fig. 5). The time of CT was most often achieved in March (25\% of outflows) and April (23\%), which highlighted the role of thaw water recharge in the aquifer water balance. Mean time of CT was 30 April but the date ranged from 23 February to 22 August. An exception was Wywierzysko Chochołowskie where the time of CT occurred in November. This was due to the fact that its subterranean catchment was partly recharged from the zone of late thaws and the recharge occurred mostly in the warmer half of the year. The second outflow with late time of CT was the spring in Skoczów, however, the results were not fully reliable due to too short period of observation (26 months).

While mean seasonality indices for the Carpathian (15.2\%) and Sudeten (15.0\%) springs were similar, the outflows located in the valleys and sub-mountain region of the Sudetes demonstrated more stable discharge than those located at higher altitudes (Fig. 6a). Analogous relationship was observed for the Carpathians and Carpathian valleys but the difference in mean time of maximum discharge was smaller. The time of $C T$ for the Sudeten springs was more homogeneous than for the Carpathian outflows (Fig. 6b). This measure seems to be less dependent on the outflow location and more on non-local factors shaping the climatic conditions in south-western Poland. The time of $C T$ in the Sudeten springs occurred on average one and a half months earlier (31 March) than in the other Carpathian springs (17 May), even without considering Wywierzysko Chochołowskie. This was only to small degree due to differences in mean altitude of the outflows, as the Carpathian ones were on average by only $30 \mathrm{~m}$ higher than those in the Sudetes. The earlier $C T$ of the Sudeten springs was caused by considerable influences of Atlantic moist and relatively war air masses that accelerated snow melting and occurrence of abundant precipitation during spring season. In the Carpathians, over half of the outflows not located within mountain massifs originated within the Podhale-Magura area. Late spring and higher total precipitation in the summer resulted in a month later time of $C T$ than in the Sudetes.

Discharge seasonality seemed independent on the outflow elevation and discharge. Both Markham's seasonality indices showed no significant correlation with spring altitude or discharge. The only significant correlation $(\mathrm{r}=0.47)$ was confirmed between discharge and time of CT. Its direction indicated that the discharge of larger outflows was typically associated with later time of $C T$ than in smaller springs, characterized by lower runoff inertia. Similar situation was observed for porous springs, in which the seasonality index was nearly three times lower than in fissure and karst outflows. However, the time of $C T$ occurred almost three weeks later than in the fissure outflows, which was also due to hydrogeological properties of the springs.

\section{Discussion}

Predominance of springs with magnitude below $1 \mathrm{dm}^{3} \cdot \mathrm{s}^{-1}$ is typical not only for the Carpathian flysch (Mocior et al., 2015) but also for crystalline rocks (Buczyński and Rzonca, 2011). Moreover, regarding to hydrogeological properties of the aquifer, fissure springs are the most common ones (Flora, 2004; Springer and Stevens, 2008). Therefore, the medians of variability measures - standard variability coefficient, Meinzer's index and Maillete's index $\left(C_{V}=60 \%, V=275 \%\right.$, $M=21.4$ ), classified majority of the outflows as springs of high variability. This was determined by the size of the drained groundwater reservoirs and the dynamics of its water table. Apart from a large number of small springs, southern Poland is abundant in permanent and poorly 

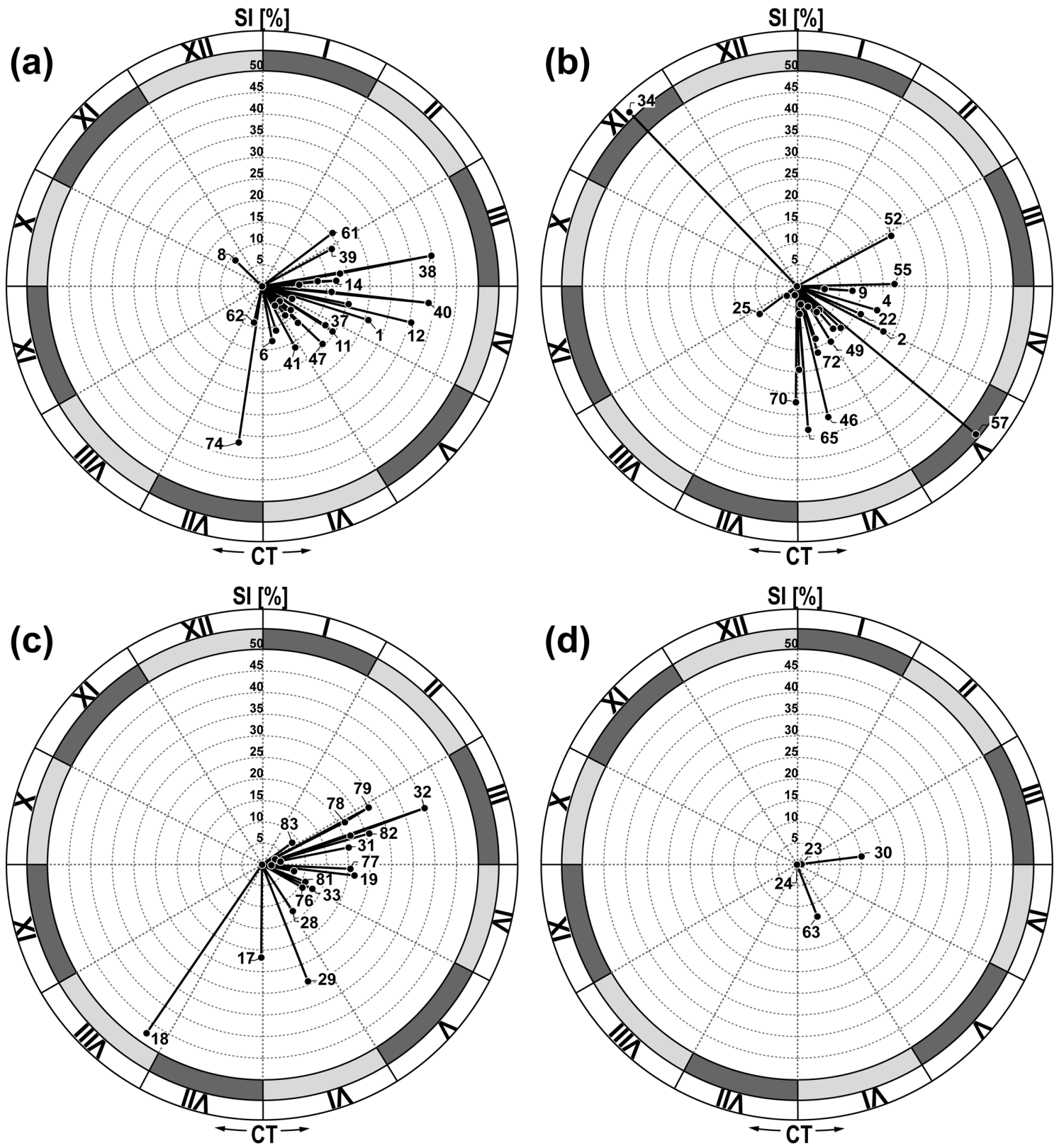

Figure 5. Interpretation of spring discharge Markham's seasonality indices in the south of Poland. The location of points indicates the time of peak discharge during the year (CT) and the length of the vectors shows seasonality power expressed by the seasonality index (SI). Numbers of springs are the same as in Fig. 1 and Table 1. a-ranges of the Carpathians; $b$-depressions, hollows and foothills of the Carpathians; $c$ ranges of Sudetes; d-depressions, hollows and foothills of the Sudetes. Source: own labor on the basis of PIG-PIB data.

variable outflows, mainly karst ones, located within Kraków-Częstochowa Upland (Chełmicki et al., 2011; Siwek and Pociask-Karteczka, 2017) and Lublin Upland (Michalczyk et al., 2008), as well as in the Tatras (Barczyk, 2003) and the Sudetes (Olichwer and Otrębski, 2016). Despite large discharge range, karst springs have deeper subterranean recharge and slower recession rate. This phenomenon was already clearly identified and described by Kovačič $(2009,2010)$ and many other authors, who discussed the time-series analysis of karst springs (Bonacci, 2001; Pulido-Bosch et al., 1995; Rahnemaei et al., 2005; Panagopoluos and Lambrakis, 2006 and Giacopetti et al., 2017). It is, however, worth mentioning that in conducive hydrogeological conditions even the fissure springs are characterized by high and sta- ble discharge (Flora, 2004). An example is the spring of the Bystrzyca Dusznicka in Zieleniec, for which the discharge variability according to Maillete was $\mathrm{M}=3.3$ and mean yield was $40.6 \mathrm{dm}^{3} \cdot \mathrm{s}^{-1}$ (Buczyński and Rzonca, 2011). It should be noted that the reliability of the obtained results is proportional to - independent of the authors - the length of available measurement series and the frequency of measurements (Table 1).

Another feature of the investigated group of springs was the relationship between their seasonality index and discharge variability. The concurrence of these metrics was already indicated by Jokiel (1997), who investigated a regime of the Carpathian springs. Variable springs had higher seasonality index. Their discharge curve was characterized by 

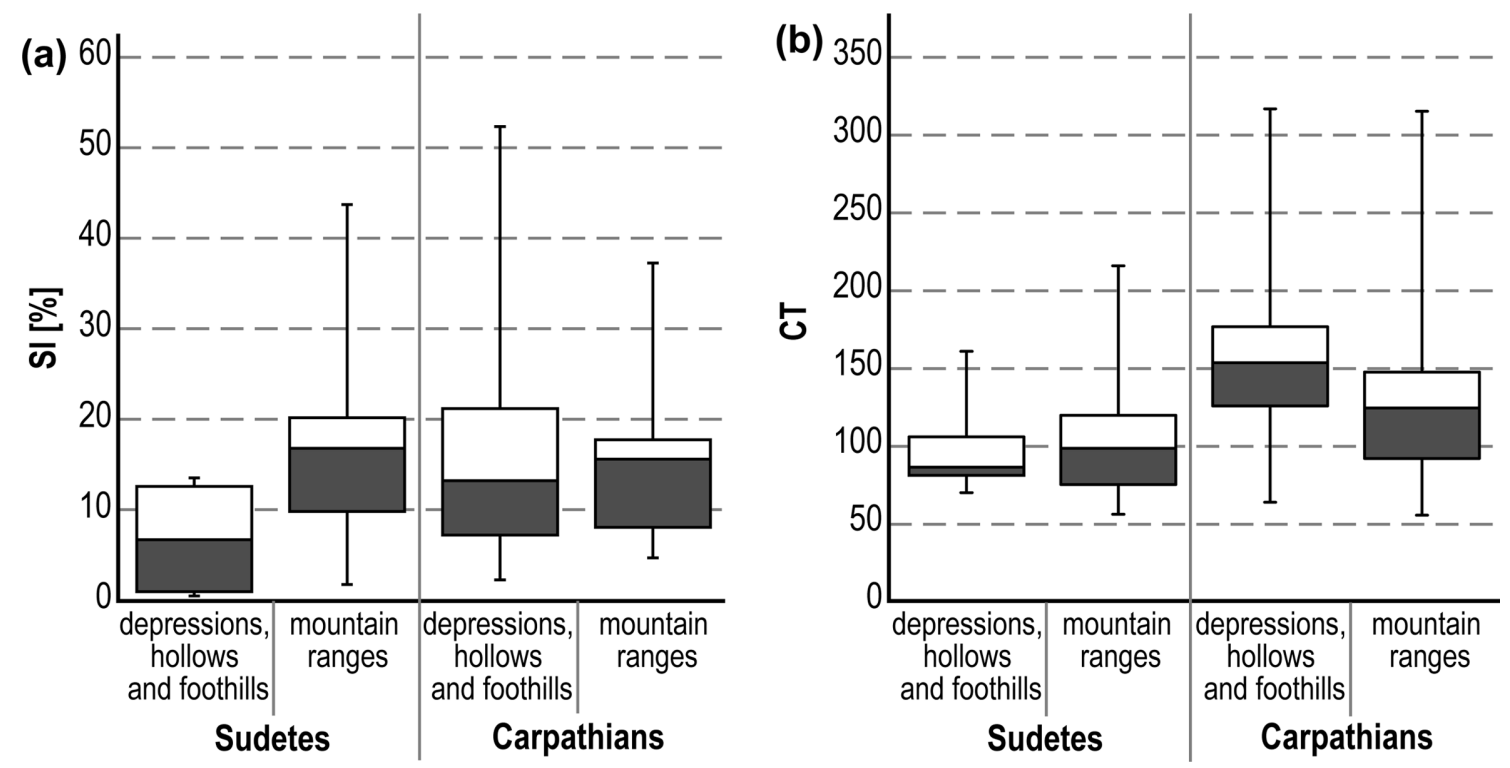

Figure 6. Main distribution characteristics of the spring discharge seasonality in the south of Poland: a-seasonality index (SI) [\%]; b-date of peak discharge (CT) [day no.]. Legend: as in Fig. 2. Source: own labor on the basis of PIG-PIB data.

a period of increased outflow during intense snow melt or precipitation related recharge. This confirmed the dependence of spring discharge variability on the size of the drained reservoir. However, variability coefficients and the seasonality index were not directly related to spring discharge volume.

The observed differences in outflow variability coefficients between mountain and sub-mountain springs were due to diverse dynamics of the mountain springs. The amount of water, outflowing from those springs, was more dependent on the distribution of precipitation than the discharge of valley and sub-mountain springs. The wider range of discharge peaks was due to abundant summer precipitation and almost no recharge in the winter (Moniewski, 2015; Pulido-Bosch et al., 1995). Response time to precipitation is usually counted here in days or even hours and is in fact limited only by the degree of rock crevassing. However, it should be remembered that the outflows from a mountain massif seemingly uniform in lithological terms may present different response to recharge. Moreover, springs located at lower elevations are often covered with colluvium or alluvium, so they are often of filtration type and may be recharged from more than one aquifer (Humnicki, 2006).

Altitude range of the investigated springs was too small (227-965 $\mathrm{m}$ a.s.l.) for the relationship between the time of $C T$ and elevation to be significant. In the extended population of central Europe's outflows, the time of $C T$ of springs located higher is later than of the springs at low altitude. Therefore, the maximum discharge of alpine outflows was usually observed a few weeks later than of Polish or Czech springs (Moniewski, 2015).

Seasonality differences are largely determined by hydrogeological type of the outflow and related aquifer response to the recharge. Regardless of the disproportion in the number of spring types, the seasonality index of porous springs was nearly three times lower than of fissure and karst springs. This was corroborated by earlier observations, made in a larger group of central Europe springs (Lo Russo et al., 2015; Moniewski, 2015), and conclusions yielded by an analysis of discharge in springs, recharged from Quaternary sediments (Chełmicki et al., 2011).

\section{Conclusions}

The discharge of southern Poland springs was characterized by considerable seasonality. It should be expected that similar relationships are observed in neighboring countries. The amount of outflowing water depended not only on current recharging conditions but mainly on hydrogeological type of the spring and its location, what is well known in general. The discharge of fissure springs that were more numerous and lesser yield than the other types is determined by the degree of aquifer crevassing, were usually small and featured much larger discharge variability than karst or porous outflows. Contrary to that, discharge variability of large karst springs might be much larger than of the other types of outflows. This is due to the karst systems are well karstified and the outflowing capacity of the spring is not somehow limited. High mountain karst springs were also characterized by a wide range of discharge extrema that was due to abundance of summer precipitation and almost no recharge in the winter. The outflows of the Sudetes featured more variable discharge than the more "continental" springs of the Carpathians. Moreover, in large springs the discharge was highly stable. The features of the continental climate became more visible towards east, which was also confirmed by a few month shift in maximum discharge in relation to precipitation observed for large fissure springs of Roztocze. Finally, seasonality of discharge was less dependent of the spring size and more on its variability, i.e. permanent springs of low variability were characterized by lower seasonality index $(S I)$ and later time of maximum discharge $(C T)$.

Above conclusions confirmed the importance of geological and meteorological conditions on the characteristics of the aquifer drained by springs. The seasonality indices can be used for many others hydrological and hydrogeological time series. In the spring discharge case they are good for the differentiation and simplify of interpretation of results in reference to the features of the geographical environment. The basic condition is to have a coherent and verified database, which is not an easy task in the case of decades of research. A conclu- 
sions based on a shorter series should be interpreted with caution. The use of additional series verification criteria (e.g., omitting discharges in extremely dry and humid years) could improve results. However, it would reduce the number of series, which in many cases could result in the exclusion of some springs from further analysis.

\section{References}

Alfaro, C. and Wallace, M., 1994, Origin and classification of springs and historical review with current applications. Environmental Geology, 24, pp. 112-124. doi: 10.1007/BF00767884

Barczyk, G., 2003, Karst and vaucluse springs from the Polish Tatra Mts. results of long-term stationary investigations. Acta Carsologica, 32, pp. 145-155. doi: 10.3986/ac.v32i1.370

Barczyk, G., Humnicki, W., and Zurawska, G., 1999, Selected hydrogeological parameters calculated for Tatric vaucluse springs. Geological Quarterly, 43, pp. 537-545.

Bartnik, A. and Moniewski, P., 2018, The content of the PGI-NRI databases in terms of research on discharges of springs in Poland. [in Polish] Przegląd Geologiczny, 66: 5, pp. 283-293.

Bartnik, A., and Tomaszewski, E., 2006, Application of the index of concentration time to assess the sensitivity of the river regime to the generation of the extreme flows in lowland catchments [in Polish]. In: Kostrzewski, A., and Czerniawska, J., (Eds.), The transformation of the geographical environment of north-western Poland. UAM, Wydz. Nauk Geogr. i Geol., Wydawnictwo Naukowe Bogucki, Poznań, Poland, pp. 137-144.

Bonacci, O., 2001, Analysis of the maximum discharge of karst springs. Hydrogeology Journal, 9, pp. 328-338. doi: 10.1007/s100400100142

Buczyński, S., and Rzonca, B., 2011, Effects of crystalline massif tectonics on groundwater origin and catchment size of a large spring area in Zieleniec, Sudety Mountains, southwestern Poland. Hydrogeology Journal, 19, pp. 1085-1101. doi: 10.1007/s10040-011-0732-3

Buczyński, S., 2017, Temporal variability of springs in catchment areas located in the Sudeten Mountains. Hydrology Research, Nov 2017, 49(3), pp. 780-793. doi: 10.2166/nh.2017.229

Chełmicki, W., Jokiel, P., Michalczyk, Z., and Moniewski, P., 2011, Distribution, discharge and regional characteristics of springs in Poland. Episodes, 34(4), pp. 244-256. http://52.172.159.94/index.php/epi/article/ view/60485

Diodato, N., Bellocchi, G., Fiorillo, F., and Ventafridda, G., 2017, Case study for investigating groundwater and the future of mountain spring discharges in Southern Italy. Journal of Mountain Science, 14, pp. 1791-1800. doi: 10.1007/s11629-017-4445-5

Dumnicka, E., Galas, J., Jatulewicz, I., Karlikowska, J., and Rzonca, B., 2012, From spring sources to springbrook: Changes in environmental characteristics and benthic fauna. Biologia, 68(1), pp. 142-149. doi: 10.2478/s11756-012-0134-6

Dynowska, I., 1986, Regional diversity of springs in Poland. [in Polish] Acta Universitatis Lodziensis. Folia Geographica Physica, 18, pp. 5-30.

Fiorillo, F., and Doglioni, A., 2010, The relation between karst spring discharge and rainfall by cross-correlation analysis (Campania, southern Italy). Hydrogeology Journal, 18, pp. 1881-1895. doi: 10.1007/s10040010-0666-1

Fiorillo, F., and Guadagno, F.M., 2012, Long karst spring discharge time series and droughts occurrence in Southern Italy. Environmental Earth Sciences, 65, pp. 2273-2283. doi: 10.1007/s12665-011-1495-9

Fiorillo, F., Petitta, M, Preziosi, E., Rusi, S., Esposito, L., and Tallini, M., 2015, Long-term trend and fluctuations of karst spring discharge in a Mediterranean area (central-southern Italy). Environmental Earth Sciences, 74, pp. 153-172. doi: 10.1007/s12665-014-3946-6

Flora, S.P., 2004, Hydrogeological characterization and discharge variability of springs in the Middle Verde River watershed, Central Arizona.
Northern Arizona University, Flagstaff, AZ, 251 p.

Giacopetti, M., Materazzi, M., Pambianchi, G., and Posavec, K., 2017, Analysis of mountain springs discharge time series in the Tennacola stream catchment (central Apennine, Italy). Environmental Earth Sciences, 76, pp. 20. doi: 10.1007/s12665-016-6339-1

Guo, Q., Wang, Y., Ma, T., and Li L., 2005, Variation of karst spring discharge in the recent five decades as an indicator of global climate change: A case study at Shanxi, northern China. Science in China, Ser. D, Earth Sciences, 48(11), pp. 2001-2010. doi: 10.1360/04yd0113

Hao, Y., Huo, X., Duan, Q., Liu, Y., Fan, Y., Liu, Y., and Yeh, T.-C.J., 2015, A Bayesian analysis of nonstationary generalized extreme value distribution of annual spring discharge minima. Environmental Earth Sciences, 73, pp. 2031-2045. doi: 10.1007/s12665-014-3552-7

Humnicki, W., 2006, The regime of the Pieniny Mts. springs in the light of limnimetric observations [in Polish]. Pieniny - Przyroda i Człowiek, 9, pp. 29-39.

Jokiel, P., 1994, Multi-annual and seasonal changes in the discharge rate of selected springs in Poland [in Polish].Wiadomości IMGW, 18(38), 4, pp. 117-130.

Jokiel, P., 1997, Basic features of the selected carpatian springs yield regime [in Polish]. Acta Universitatis Lodziensis. Folia Geographica Physica, 2, pp. 27-43. http://hdl.handle.net/11089/2898

Katsanou, K., Maramathas, A., and Lambrakis, N., 2015, Simulation of Karst Springs Discharge in Case of Incomplete Time Series. Water Resources Management, 29, pp. 1623-1633. doi: 10.1007/s11269014-0898-2

Kovačič, G., 2009, Hidrologija kraškega izvira Malenščica in njegovega hidrografskega zaledja [Hydrology of the Malenščica karst spring and its catchment]. 329f. Tese (Doutorado) - Universidade de Primorska, Faculdade de Humanidades, [in Slovenian] https://repozitorij.upr.si/ Dokument.php?id=11886\&lang $=$ slv

Kovačič, G., 2010, Hydrogeological study of the Malenščica karst spring (SW Slovenia) by means of a time series analysis. Acta Carsologica 39(2), pp. 201-215. doi: 10.3986/ac.v39i2.93

Kresic, N., and Stevanovic, Z., 2009, Groundwater Hydrology of Springs: Butterworth-Heinemann. doi: 10.1016/B978-1-85617-502-9.00014-1

Kř́ž, H., 1973, Processing of Results of Observations of Spring Discharge. Groundwater, 11(5), pp. 1-14. doi: 10.1111/j.1745-6584.1973.tb02981.x

Łajczak, A., 2012, Water circulation and chemical denudation within the upper Skawica River flysch catchment, Western Carpathian Mountains. Zeitschrift für Geomorphologie, Supplementary Issue, 56(1), pp. 69-86. doi: 10.1127/0372-8854/2012/S-00073

Liu, Y., Wang, B., Zhan, H., Fan, Y., Zha, Y., Hao, Y., 2017, Simulation of Nonstationary Spring Discharge Using Time Series Models. Water Resources Management, 31, pp. 4875-4890. doi: 10.1007/s11269-017$1783-6$

Lo Russo, S., Amanzio, G., Ghione, R., and De Maio, M., 2015, Recession hydrographs and time series analysis of springs monitoring data: application on porous and shallow aquifers in mountain areas (Aosta Valley). Environmental Earth Sciences, 73, pp. 7415-7434. doi: 10.1007/ s12665-014-3916-z

McCann, T., 2008a, The Geology of Central Europe. Vol. 1: Precambrian and Palaeozoic. Geological Society, London. doi: 10.1144/CEV1P

McCann, T., 2008b, The Geology of Central Europe. Vol. 2: Mesozioc and Cenozoic. Geological Society, London. doi: 10.1144/CEV2P

Markham, C.G., 1970, Seasonality of precipitation in The United States. Annals of the Association of American Geographers, 60, pp. 593-597. doi: 10.1111/j.1467-8306.1970.tb00743.x

Memon, B.A., 1995, Quantitative analysis of springs. Environmental Geology, 26, pp. 111-120. doi: 10.1007/BF00768324

Michalczyk, Z., Chmiel, S., Głowacki, S., and Zielińska, B., 2008, Changes of spring's yield of Lublin Upland and Roztocze Region in 1998-2008. Journal of Water and Land Development, 12, pp. 113-125. doi: 10.2478/v10025-009-0009-6

Mocior, E., Rzonca, B., Siwek, J., Plenzler, J., Placzkowska, E., Dabek, 
N., Jaskowiec, B., Potoniec, P., Roman, S., and Zdziebko, D., 2015, Determinants of the distribution of springs in the upper part of a flysch ridge in the Bieszczady Mountains in southeastern Poland. Episodes, 38(1), pp. 21-30. http://52.172.159.94/index.php/epi/article/view/63802

Moniewski, P., 2015, Seasonal Variability of Discharge from Selected Springs in Central Europe. Episodes, 38(3), pp. 189-196. http://52.172.159.94/ index.php/epi/article/view/79714

Olichwer, T., and Otrebski, A., 2016, Groundwater renewable resources in karst areas, the case of the Kleśnica River basin (Sudety Mountains, Poland). Geoscience Records, 2(1), pp. 17-23. doi: 10.1515/georec2016-0003

Panagopoluos, G., and Lambrakis N., 2006, The contribution of time series analysis to the study of the hydrodynamic characteristics of the karst systems: Application on two typical karst aquifers of Greece (Trifilia, Almyros Crete). Journal of Hydrology, 329, pp. 368-376. doi: 10.1016/j.jhydrol.2006.02.023

Pulido-Bosch, A., Padilla, A., Dimitrov, D., and Machkova, M., 1995, The discharge variability of some karst springs in Bulgaria studied by time series analysis. Hydrological Sciences Journal, 40, pp. 517-532. doi: 10.1080/02626669509491436

Rahnemaei, M., Zare, M., Nenatollahi, A.R., Sedghi, H., 2005, Application of spectral analysis of daily water level and spring discharge hydrographs data for comparing physical characteristics of karst aquifers. Journal of Hydrology, 311, pp.106-116. doi: 10.1016/j.jhydrol.2005.01.011

Siwek, J., 2013, Springs yield [in Polish]. In: Siwek, J., and Bascik, M., (Eds.) The natural and anthropogenic changes of springsin the Krakowsko-Wieluńska and Miechowska Uplandsand the role of springs in the naturaland cultural landscapes. IGiGP, UJ, Kraków, pp. 45-58.

Siwek., J., and Pociask-Karteczka, J., 2017, Springs in South-Central Poland the changes and threats. Episodes, 40, pp. 38-46. doi: 10.18814/epiiugs/2017/v40i1/017006

Solon, J., Borzyszkowski, J., Bidłasik, M., Richling, A., Badora, K., Balon, J., Brzezińska-Wójcik, T., Chabudziński, Ł., Dobrowolski, R., Grzegorczyk, I., Jodłowski, M., Kistowski, M., Kot, R., Krąż, P., Lechnio, J., Macias, A., Majchrowska, A., Malinowska, E., Migoń, P., Myga-Piątek, U., Nita, J., Papińska, E., Rodzik, J., Strzyż, M., Terpiłowski, S., and Ziaja, W., 2018, Physico-geographical mesoregions of Poland: Verification and adjustment of boundaries on the basis of contemporary spatial data. Geographia Polonica, vol. 91, no. 2, pp. 143-170. doi: 10.7163/ GPol.0115

Springer, A.E., and Stevens, L.E., 2008, Spheres of discharge of springs. Hydrogeology Journal 17, pp. 83-93. doi: 10.1007/s10040-008-0341-y

Tarafdar, S., 2013, Understanding the dynamics of high and low spring flow: a key to managing the water resources in a small urbanized hillslope of Lesser Himalaya, India. Environmental Earth Sciences, 70, pp. 2107-2114. doi: 10.1007/s12665-011-1493-y

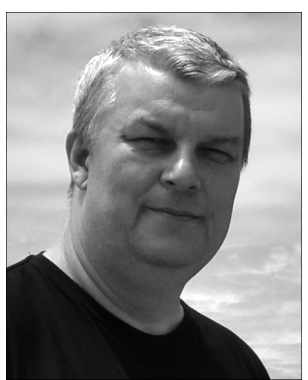

Adam Bartnik Ph.D. is a hydrologist at the University of Lodz, Department of Hydrology and Water Management. His research interests involve three main subject areas: the widely understood spring water circulation, application of geostatistics and GIS in hydrology and the impact of anthropopressure at quality and quantity of water. He is author of over 80 peer reviewed papers, maps, book chapters and books and supervisor of Master and Bachelor students.

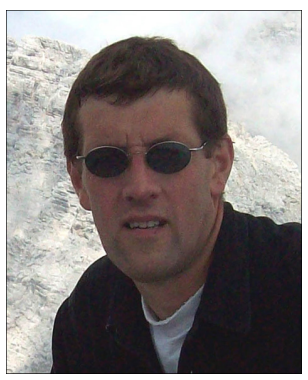

Piotr Moniewski Ph. D. is an employee in the Chief Inspektorate of Environmental Protection, Central Research Laboratory, Department in Lodz, Poland. His interests focus on groundwater outflows, water resources and hydrological measurement techniques, and human impact on air and water quality in rural and urbanized drainage basins. He has published over 50 research papers in international and Polish journals. 